Rabaska

Revue d'ethnologie de l'Amérique française

\title{
Prix, honneurs et distinctions
}

\section{Élise Bégin}

Volume 16, 2018

URI : https://id.erudit.org/iderudit/1051338ar

DOI : https://doi.org/10.7202/1051338ar

Aller au sommaire du numéro

Éditeur(s)

Société québécoise d'ethnologie

ISSN

1703-7433 (imprimé)

1916-7350 (numérique)

Découvrir la revue

Citer ce document

Bégin, É. (2018). Prix, honneurs et distinctions. Rabaska, 16, 222-227.

https://doi.org/10.7202/1051338ar

Ce document est protégé par la loi sur le droit d'auteur. L’utilisation des services d'Érudit (y compris la reproduction) est assujettie à sa politique d'utilisation que vous pouvez consulter en ligne.

https://apropos.erudit.org/fr/usagers/politique-dutilisation/
Cet article est diffusé et préservé par Érudit.

Érudit est un consortium interuniversitaire sans but lucratif composé de l’Université de Montréal, l'Université Laval et l'Université du Québec à Montréal. Il a pour mission la promotion et la valorisation de la recherche. https://www.erudit.org/fr/ 


\title{
Prix, honneurs et distinctions
}

\author{
ÉLISE BÉGIN \\ Société québécoise d'ethnologie
}

\section{Prix Gérard-Morisset 2017}

En 2017, la plus haute distinction du gouvernement du Québec dans le domaine de la culture a été décernée à Jean Simard pour souligner sa contribution à la connaissance et à la mise en valeur du patrimoine québécois tout au long de sa carrière. La cérémonie s'est déroulée le $1^{\text {er }}$ novembre dans la prestigieuse salle du Conseil législatif à l'Hôtel du Parlement, en présence de plusieurs membres de la Société québécoise d'ethnologie ainsi que des membres de la famille. Jean Simard a œuvré pour la connaissance et la compréhension de l'ethnologie québécoise au cours des 45 dernières années à travers des études et des recherches-actions, plus spécialement dans le domaine du patrimoine religieux. Le caractère pionnier de ses recherches, marqué en particulier par des inventaires méthodiques, a fait progresser la réflexion, la conscience et la défense d'un domaine que bien peu de ses prédécesseurs avaient étudié avec autant d'envergure et d'esprit. Son œuvre est reconnue non seulement au Québec mais aussi à l'extérieur du Québec, notamment au Canada, aux États-Unis, au Mexique et en Europe. Voir à la rubrique Portrait de ce volume « Jean Simard, le philosophe ethnologue » par René Bouchard.

\section{Bourse d'études Luc-Lacourcière 2017}

L'ACEF a décerné cette année sa bourse d'études Luc-Lacourcière 2017 à Jennifer Boivin, doctorante à l'Université d'Alberta. Le prix est décerné à un étudiant ou une étudiante membre de l'ACEF qui a fait preuve d'excellence en ethnologie ou études du folklore, qui a complété sa première année d'études supérieures dans une université canadienne et qui est toujours inscrit aux études dans cette discipline. L'ACEF a établi un fonds qui entretiendra sa bourse d'études qui commémore la nomination du professeur Luc Lacourcière à la première chaire de folklore établie dans une université canadienne ainsi que son engagement dans l'avancement de la discipline au Canada et à l'étranger. 


\section{Prix Mnémo 2017}

Le Prix Mnémo vise à reconnaître, à récompenser et à mettre en valeur une œuvre jugée remarquable dans le domaine de la documentation ou de la recherche relative à la danse, la musique, la chanson et le conte traditionnels des francophones d'Amérique. Cette année, ce $19^{\mathrm{e}}$ prix a été remis à Robert Bouthillier pour son CD-DVD Temporel / Intemporel. La cérémonie a eu lieu le 2 décembre dans le cadre des 12 heures $d u$ chant organisées par l'Atelier de chant traditionnel de Québec, en collaboration avec le CvPv ès trad. Temporel / Intemporel permet non seulement de suivre le chemin d'une chanson à travers plusieurs versions de différents informateurs, mais aussi d'entendre l'interprétation offerte par M. Bouthillier et le chemin parcouru par chaque chanson depuis sa cueillette. Rabaska a publié un compte rendu de cette production (vol. 15, 2017, p. 284-285).

\section{Prix Carrière 2017 de la Société des musées du Québec}

Le prix Carrière souligne la contribution exceptionnelle et continue d'un membre de la Société des musées du Québec à la muséologie québécoise. Le lauréat de cette année est Philippe Dubé, jusqu'à tout récemment professeur au département des sciences historiques à l'Université Laval. Depuis les années 1980, la carrière de Philippe Dubé est marquée par sa passion pour la muséologie et son approche ethnohistorique. D'abord conservateur au Musée régional Laure-Conan (aujourd'hui Musée de Charlevoix), il présente une exposition sur la villégiature dans Charlevoix qui mérite un prix en tourisme culturel et il signe en 1988 l'exposition inaugurale « Mémoires » au Musée de la Civilisation. Nommé professeur à l'Université Laval, il fonde et dirige le programme de deuxième cycle en muséologie où il dirigera plus de 200 étudiants à la maîtrise professionnelle, 15 doctorats et plus de 10 aspirants à la maîtrise en recherche dans les domaines de l'ethnologie, de l'histoire, de l'archéologie et de l'histoire de l'art. Il applique la méthodologie du terrain pour recueillir ses données et s'intéresse ensuite à la caractérisation d'une micro-muséologie en contexte régional. En 2004, il conçoit le Laboratoire de muséologie et d'ingénierie de la culture, le Lamic, orienté vers l'étude des modalités de transmission en contexte muséal qui ouvre ses portes en 2007. C'est dans ce cadre qu'il a réalisé la reconstruction numérique 3D de la chapelle des Ursulines de Québec ainsi que l'espace de vie du monastère, afin de reproduire l'esprit du lieu et de mettre en valeur l'héritage des religieuses. En 2008, il a coscénarisé le Moulin à images, fresque historique conçue par Robert Lepage projetée sur les silos à grains dans le Vieux-Port de Québec. Ses nombreuses publications et ses conférences ont permis de diffuser ses 
résultats de recherche dans le développement de nouvelles pratiques de la muséologie populaire et de la mise en exposition.

\section{Prix Encadrement aux cycles supérieurs de l'Université Laval 2017}

Le 12 novembre 2017, Laurier Turgeon a été lauréat du prix Encadrement aux cycles supérieurs pour souligner l'excellence de son enseignement à l'Université Laval. Professeur au département des sciences historiques de la Faculté des lettres et des sciences humaines depuis 1985 en ethnologie et patrimoine, Laurier Turgeon offre un encadrement riche et personnalisé à ses étudiants de la maîtrise au post-doctorat ainsi qu'à des stagiaires d'été au premier cycle en ethnologie. Polyvalent et reconnu pour son expertise, Laurier Turgeon encadre un nombre impressionnant d'étudiants en les accompagnant avec sensibilité et rigueur dans l'avancement de leur recherche. Il aide les étudiants à exploiter leur plein potentiel de leur passion, en leur confiant par exemple une pleine autonomie quant au choix de leur sujet de recherche, ce qui témoigne de sa grande capacité d'adaptation. Il se préoccupe de leurs objectifs personnels et professionnels futurs et entretient des relations significatives avec plusieurs diplômés au-delà de leurs études. Il met en place des conditions stimulantes et facilitantes pour l'avancement de leur processus et leur fait profiter de son riche réseau de contact à l'échelle nationale et internationale pour faciliter leur insertion professionnelle. Le nombre important de ses étudiants obtenant des bourses et des prix pour leurs recherches ainsi que son engagement à long terme démontrent à quel point monsieur Turgeon offre un encadrement de qualité remarquable.

\section{Chaire de recherche stratégique de l'UQÀm en gouvernance des musées}

À la suite de la création par l'Université du Québec à Montréal de quatre nouvelles chaires pour un mandat de trois ans, une toute nouvelle chaire stratégique, consacrée à la gouvernance des musées et attribuée à un seul titulaire par voie de concours, échoit, avec tous les honneurs qui vont avec cette fonction éminente, à Yves Bergeron, ancien directeur de l'Institut du patrimoine et professeur titulaire de muséologie et de patrimoine à l'UQÀM. Cette nouvelle chaire sur la gouvernance des musées répond à la recommandation émise par l'UNESCO au sujet de l'un des enjeux les plus sensibles du XXI iècle : préserver la diversité culturelle au regard des collections muséales, de leur protection et de leur pérennité. La chaire adoptera une approche originale pour l'étude et la formation en muséologie, fondée sur la convergence de la muséologie, de la gestion et du droit. Ce faisant, elle développera des outils novateurs de gestion adaptés pour le milieu professionnel. Yves Bergeron, grand spécialiste 
du domaine, dont la feuille de route à cet égard est impressionnante, est qualifié et bien placé pour relever ce mandat.

\section{Le Billochet du jongleur 2018}

Après avoir remporté la Médaille Luc-Lacourcière (1991) puis la Médaille Marius-Barbeau (2013), Jean-Pierre Pichette s'est mérité le 21 février 2018 le prestigieux prix du Billochet du jongleur. Institué en hommage au père Germain Lemieux, celui-ci représente la plus haute récompense de l'Ontario francophone en matière de patrimoine. Remis depuis 2002 par le Centre franco-ontarien de folklore, ce prix témoigne du mérite particulier d'une personne qui aura fait une contribution exceptionnelle pour la reconnaissance, la sauvegarde ou la mise en valeur du patrimoine oral francoontarien. Voué à l'enseignement et à la recherche, Jean-Pierre Pichette a eu une carrière marquée par de grandes réalisations que l'Université de Sudbury a voulu reconnaître en lui attribuant en 2014 un doctorat honoris causa pour l'ensemble de son œuvre. Il a participé à l'essor des études franco-ontariennes et a constitué des archives de folklore d'une richesse inestimable au cours de ses 23 années dans l'Ontario francophone. Des ouvrages de sa plume ou issu de sa direction éclairée, des projets d'envergure seront devenus des références incontournables pour l'étude de l'Ontario français, tels Le Répertoire ethnologique de l'Ontario français (1992), Habiter le pays. L'Inventaire du patrimoine en Ontario français (2001) et le Dictionnaire des écrits de l'Ontario français (1613-1993) (2010). Il aura joué de plus un rôle exemplaire d'animateur intellectuel en fondant et dirigeant des revues de haut calibre, comme la Revue du Nouvel-Ontario (1982-1988) ou les Cahiers Charlevoix (12 vol. de 1995 à 2018).

\section{Concours " Votre Sherbrooke "}

Le prix du concours « Votre Sherbrooke», organisé par les Éditions du Péricarde, est attribué cette année à Bernard Genest pour son texte « L'Oncle Valmore » qui raconte la vie de l'un de ses oncles, le docteur Valmore Olivier. Les participants à ce concours devaient soumettre un texte qui raconte une histoire personnelle liée à l'histoire de la ville de Sherbrooke. Jacques Couture, éditeur de la maison d'édition, souligne que c'est unanimement que les membres du jury ont choisi ce récit, apprécié pour la qualité d'écriture de l'auteur, qui traverse le $\mathrm{xx}^{\mathrm{e}}$ siècle, son histoire et ses traditions locales. Il ajoute : " le récit de Bernard Genest aborde toute une série de thèmes qui décrivent avec finesse de nombreux caractères spécifiques de la ville Reine des Cantons ». De sa carrière militaire à sa vie professionnelle comme chirur- 
gien-dentiste, l'histoire de l'oncle Valmore souligne avec beaucoup d'intérêt sa contribution à la santé publique lors de son passage à l'Unité sanitaire de Sherbrooke dans les années 1950, sa participation à la naissance de la société Saint-Jean-Baptiste locale, son engagement envers la défense du fait français à travers son militantisme au sein du Bloc populaire canadien, un mouvement nationaliste préfigurant le Parti Québécois.

\section{Médaille Marius-Barbeau 2018}

La médaille Marius-Barbeau, décernée par l'Association canadienne d'ethnologie et de folklore (ACEF), a été remise conjointement à Georges Arsenault et John Cousins pour leur contribution au cours de leur carrière à la collection, à l'étude, à la diffusion et à la popularisation du folklore dans la province de l'Île-du-Prince-Édouard, voire même au-delà. Georges Arsenault et John Cousins, nés et ayant étudié dans leur communauté, se sont investis à l'étude du folklore, le premier sur les traditions de sa communauté acadienne et le second sur le paysage de West Prince, l'île du Prince-Édouard. La contribution scientifique de Georges Arsenault à l'histoire et au patrimoine acadien est incontournable, et ses nombreuses publications, conférences à titre de chercheur, animateur, conseiller, journaliste et conservateur lui ont valu plusieurs distinctions, telles que la nomination à l'Ordre de l'île-du-Prince-Édouard et à l'Ordre du Canada (2003 et 2016), les diplômes honoraires de l'Université de l'Île-du-Prince-Édouard (2004) aussi bien que de l'Université de Moncton (2017), le prix d'honneur du Musée de l'Île-du-Prince-Édouard pour ses contributions au cours de sa carrière au patrimoine de sa province et sa nomination à l'Ordre des arts et des lettres de la République Française. John Cousins, représentant du folklore de langue anglaise de son île, est devenu une personnalité emblématique de la province, où il collectionne, célèbre, chante, promeut et étudie le patrimoine depuis une cinquantaine d'années. Il a participé en tant qu'interprète local de chansons à de nombreux festivals de folklore de l'île du Prince-Édouard.

\section{Salon des antiquaires de Kingsey-Falls}

Lors d'un grand banquet regroupant près de 300 convives le 9 juin 2018, les antiquaires et les passionnés de biens culturels réunis pour trois jours à KingseyFalls, aux Jardins Marie-Victorin, lors du premier Salon des antiquaires de Kingsey-Falls, ont rendu un vibrant hommage à notre collègue Michel Lessard, invité d'honneur à cette rencontre. Les professionnels du commerce des objets anciens voulaient ainsi souligner la contribution exceptionnelle du professeur Lessard à la connaissance de notre culture matérielle par ses livres, ses films et son action depuis 40 ans. Près de 5000 visiteurs ont franchi les 
guichets de ce salon qui reviendra l'an prochain dans le même cadre exceptionnel. Les antiquaires et les collectionneurs attribuent aux publications de Michel Lessard une grande valeur de référence en culture matérielle, en raison de sa connaissance étendue et savante des collections privées issues de ce milieu. Sa Nouvelle Encyclopédie des antiquités du Québec, vendue à près de 20000 exemplaires, est un indice probant de la notoriété de notre collègue à cet égard et de l'heureuse rencontre d'un universitaire et des professionnels en matière de patrimoine. 